\title{
Gemeinsamkeiten und strukturelle Unterschiede der Corporate und Nonprofit Governance
}

Completed Staffwork; Corporate Governance; Ehrenamt; Nonprofit Governance; Typenabhängige Governance-Ansätze; Vorstandsstrukturen

Die unterschiedlichen Führungs- und Organisationsstrukturen in NonprofitOrganisationen (NPO) erfordern angepasste Governance-Massnahmen. In der Folge werden einige der der Best Practice-Prinzipien des Swiss Code of Corporate Governance für Aktiengesellschaften mit entsprechenden Gestaltungsempfehlungen der NPOManagement-Lehre verglichen. Dabei werden Gemeinsamkeiten und Unterschiede in der Corporate und der Nonprofit Governance herausgearbeitet sowie typenspezifische Probleme aufgezeigt und daraus entsprechende Handlungsempfehlungen für eine wirkungsvolle Nonprofit Governance abgeleitet.

\section{Der Corporate Governance-Ansatz}

Die Debatte um Governance und Verantwortlichkeit wird in allen drei Sektoren - Staat, Wirtschaft und NPO - geführt und beschäftigt gleichermaßen Wissenschaftler und Praktiker.

Der Begriff der Corporate Governance als Oberbegriff für die Regelung der Unternehmensüberwachung und die strategische Unternehmensführung geht auf das Jahr 1992 und die "Cadbury-Kommission“ zurück, welche in England einen Bericht und einen Massnahmenkatalog zur Gestaltung der Unternehmensführung und -überwachung verfasste. ${ }^{1}$ Missmanagement in Unternehmungen ist die Folge eines Steuerungs- und Kontrollvakuums zwischen Aktionären/Anlegern und Verwaltungs-/Aufsichtsräten (Boards) auf der einen und dem Management (Geschäftsführung, Chief Executive Officer (CEO), Direktorium) auf der anderen Seite. Die im Prinzip zur Vertretung der Aktionärinteressen beauftragten Verwaltungsräte haben der Experten- und Informationsmacht des Managements zu wenig entgegenzusetzen. ${ }^{2}$ Mit der Entscheidungsvorbereitung betraut legt der CEO dem (nebenamtlichen und oft rein fachlich qualifizierten) Verwaltungsrat Strategievarianten vor, deren Entstehung (mit zahlreichen Vorentscheidungen, Bewertungen) vielfach nicht nachvollzogen werden kann und deren Folgen oft nicht absehbar sind. CEO praktizieren ein sog. Completed Staff-work. Darunter versteht man eine Vorgehensweise, welche die Verwaltungsräte zu Genehmigungsgremien degradiert, denen die Möglichkeit zum Mitprägen dieser oder gar Durchsetzen anders lautender Strategien weitgehend ver-

1 Vgl. Cadbury-Report (1992). Vgl. ausführlich zur Entstehungsgeschichte Brönnimann (2003), S. 57 ff.

2 Vgl. Jenson/Meckling (1976). 
sperrt bleibt, was sie jedoch von ihrer Verantwortlichkeit gegenüber den Aktionären keineswegs entbindet.

Hinter dieser faktischen Allmacht des Managements steckt zudem ein Konflikt, den die Genossenschaftswissenschaft als ein Auseinanderklaffen von Managementmotivation und Mitgliederinteressen thematisiert hat. ${ }^{3}$ Manager wollen Wachstum, Größe bis zur Marktdominanz und handeln in diesem Streben gegen die Interessen der Träger (Genossenschafter, Aktionäre), dies nicht zuletzt dann, wenn sie die Erfolge kurzfristig anstreben und die längerfristige Substanzerhaltung oder gar die Weiterentwicklung der Organisation gefährden.

Diesem Machtungleichgewicht will der Corporate Governance-Ansatz in drei Bereichen entgegen wirken:

1) Steigerung der ,Qualität“ des Verwaltungsrates in seiner Steuerungs- und Kontrollfunktion,

2) Entwicklung entsprechender, tauglicherer Managementinstrumente,

3) Schaffen vermehrter Transparenz, für alle Beteiligten und Betroffenen.

Im Swiss Code of Best Practice wird ein weitgehender Massnahmenkatalog formuliert, um diese Corporate Governance Ziele zu erreichen. ${ }^{4}$ Auf ausgewählte Ziele und Massnahmen wird im Rahmen der Prüfung der Anwendbarkeit dieses Ansatzes im NonprofitSektor näher eingegangen.

\section{Die Ehrenamt-Hauptamt-Problematik in NPO}

Die freiwillig-ehrenamtliche Tätigkeit in den dem Hauptamt übergeordneten Führungsorganen ist ein existenzielles Charakteristikum von NPO. Das Zusammenwirken von Ehrenamtlichen und Hauptamtlichen ist jedoch durch ein strukturelles Spannungsfeld gekennzeichnet, welches unter Umständen das Wohl der gesamten Organisation bedroht. Die Ehrenamtlichkeit der mitwirkenden Mitglieder steht vielerorts im Gegensatz zur vollamtlichen Professionalität und dem Selbstverständnis der Geschäftsführung. Dieses Spannungsfeld äussert sich einerseits durch die Abhängigkeit der ehrenamtlichen Amtsträger vom Geschäftsbetrieb und andererseits in der Machtbeziehung zwischen den beiden Parteien. Die professionelle Geschäftsführung besitzt gegenüber den ehrenamtlichen Mitgliedern einen Wissens-, Erfahrungs- und Informationsvorsprung. Je mehr sich das Machtgleichgewicht vom ehrenamtlichen System zur professionellen Geschäftsführung verlagert, desto bedeutsamer wird auch das Thema der Nonprofit Governance. Dabei sind unter Einbeziehung der vielfältigen Austauschpartner (Stakeholder) auch spezifische Probleme von Drittleistungs- und Eigenleistungs-NPO näher zu beachten.

In jüngster Zeit häufen sich auch in der Schweiz Meldungen über Missmanagement in Nonprofit-Organisationen. Dabei ist im Einzelfall zu untersuchen, ob nicht ein eigentliches Nicht-Management vorliegt, im Sinne einer weitgehenden Absenz von Manage-

3 Vgl. Vierheller (1983).

4 Vgl. Böckli (2002). 
ment-Know-how, nicht zuletzt auf Seiten der ehrenamtlichen (Miliz-)-Organe (Vereinsvorstände, Stiftungsräte). Es stellt sich somit die aktuelle Frage, ob grundsätzlich Governance als dringende Forderung auch an NPO zu richten sei, und wenn ja, inwieweit der profitorientierte Corporate Governance-Ansatz auch einheitlich auf alle NonprofitOrganisationen übertragbar ist.

NPO konstituieren sich mehrheitlich in den Rechtsformen des Vereins oder der Stiftung, teilweise als Genossenschaft. Sie werden satzungsmässig von ehrenamtlichen Gremien (Vorstände, Stiftungsräte) geleitet, in Vereinen und Genossenschaft im Rahmen der Beschlüsse von Mitglieder-/Delegiertenversammlungen. Größere NPO mit einem breiten Leistungsangebot verfügen zumeist über einen Betrieb (Geschäftsstelle, Institut, Heime usw.) mit hauptamtlich angestellten Mitarbeitenden, darunter die leitende Geschäftsführung, welche dem Ehrenamtsgremium unterstellt ist.

Je nach Ausgestaltung einer NPO kann die Zusammenarbeit von Ehrenamtlichen und Hauptamtlichen sehr komplex und konfliktbeladen sein, und zwar unabhängig von der wirtschaftlichen Lage, in der sich die Organisation befindet. Es kann deshalb nicht erstaunen, dass das oben kurz skizzierte Verhältnis zwischen Verwaltungsrat und CEO, welches zu Beginn der 1990er Jahre zur Corporate Governance Debatte geführt hat, in NPO in analoger Weise permanent ein schwieriges Verhältnis war und ist. ${ }^{5}$ Anders als die zumeist gut bezahlten Verwaltungsräte sind Ehrenamtliche freiwillig tätig mit meist nur geringen Entschädigungen, was die Problematik des Ungleichgewichtes noch verstärkt. Ehrenamtliche sind - als „Laien“ - bezüglich Zeit, Sachverstand und Information der hauptamtlichen Geschäftsführung gegenüber im Nachteil, sie verfügen eher über geringes organisationsspezifisches Management-Know-how und sind damit dem Completed Staff-work der Geschäftsführung in hohem Masse ausgesetzt. Diese hält sich oftmals bedeckt, versorgt den Vorstand nur spärlich und auf eigenen Vorteil bedacht mit Informationen. Die übrigen Austauschpartner wie Mitglieder, Kunden etc. sind über die Aktivitäten der Geschäftsführung kaum informiert.

Im Gegenzug sind die ehrenamtlichen Führungsorgane ihrerseits nicht automatisch an einem effizienten und effektiven Management von NPO interessiert, denn die sie motivierenden Anreize zu Amtsübernahme in Form von Gewinn an Einfluss, Reputation und sozialem Kapital durch die Zugehörigkeit zu Netzwerken bestehen in relativer Unabhängigkeit vom erfolgreichen Wirken der NPO. Seibel spricht gar von möglichen inversen Anreizstrukturen. Diese führen dazu, dass Vorstandsmitglieder, indem sie der Organisation Ressourcen beschaffen und dadurch die Abhängigkeitsbeziehung der NPO verstärken, in erster Linie die Universalressource Macht zu maximieren trachten. ${ }^{6}$ Auch Houle warnt vor dem Amtsmissbrauch der Mitglieder der Aufsichtsorgane zu egoistischen Zwecken. $^{7}$ Duca bemängelt die Tatsache, dass sich Board-Mitglieder immer wieder in das operative Geschäft einmischen und dabei ihre eigentlichen Führungs- und Überwachungsaufgaben sträflich vernachlässigen. ${ }^{8}$

\footnotetext{
Vgl. Schwarz (1996), S. 524.

Vgl. Seibel (2002), S. 22.

Vgl. Houle (1997), S. 17 f.

Vgl. Duca (1996) S. 12 f.
} 
Die Virulenz der Ehrenamt-Hauptamt-Problematik, die häufig fehlenden NPO-adäquaten Management-Instrumente und die ungenügende Informationspolitik sind seit Jahren eine Herausforderung an die NPO-Management-Lehre, taugliche Problemlösungen in diesen Bereichen für die Praxis bereitzustellen. Das bedeutet zugleich, dass die Philosophie und die genannten Ziele der Corporate Governance für NPO keine grundlegende Innovation beinhalten. Insbesondere die angelsächsische Literatur befasst sich seit langem mit Fragen der Nonprofit Governance und der Rolle von Aufsichtsorganen (Boards). ${ }^{9}$ Im deutschsprachigen Raum hat sich insbesondere die Genossenschaftswissenschaft ${ }^{10}$ recht früh mit dieser Problematik auseinandergesetzt. Inzwischen wurden auch hier allgemeingültige Ansätze für alle NPO-Typen entwickelt. ${ }^{11}$

\section{Die Rolle der Aufsichtsorgane (Boards)}

Als wichtigste Aufgaben von Vorständen und Stiftungsräten werden zum einen Meinungs- und Willensbildung und zum anderen die Kontrolle des operativen Betriebes betrachtet. ${ }^{12}$ Zusammensetzung, Größe und Struktur entscheiden dabei über die Effektivität und Effizienz ehrenamtlicher Organe. ${ }^{13}$ Von ihnen wird die Führung der Organisation durch die Vorgabe einer Politik und Strategie verlangt. ${ }^{14}$ Dabei sollen die Mitglieder dieses Gremiums auch Kontakte zu externen Meinungsmachern sowie Politik und Staat entwickeln und gleichzeitig Zielkonflikte zwischen der NPO-Mission und der ökonomischen Wirtschaftlichkeit überbrücken helfen. ${ }^{15}$

Als „Aufsichts-“Organ obliegt es dem Vorstand zudem, die Aktivitäten der Organisation zu überwachen und zu bewerten. Die Schwierigkeit dieser Aufgabe liegt darin, dass sich kaum messbare Kriterien definieren lassen, anhand derer man den Erfolg einer NPO beurteilen kann. Hier sind die Vorstandsmitglieder oftmals auf die Aussagen des Managements angewiesen. Die Qualität ihrer Kontrolle der operativen Stellen hängt deshalb häufig davon ab, wer in der Beziehung zwischen Geschäftsführung und Vorstand die dominante Rolle übernimmt. ${ }^{16}$

Den Versuch einer Differenzierung der Governance-“Stile” haben erstmals Alexander/Weiner vorgenommen, indem sie zwischen Corporate und Philanthropic Governance unterscheiden. Ihre Kriterien beziehen sich sowohl auf die Board-Struktur, die Arbeitsteilung zwischen Vorstand und Geschäftsführung sowie die Arbeitsgestaltung der BoardMitglieder, wie in Tabelle 1 nachzuvollziehen ist.

9 Vgl. Drucker (1990); Murray u.a. (1992); Holland (2002); Saidel/Harlan (1998).

10 Vgl. Vierheller (1983).

11 Vgl. Schwarz (2005), S. 225 ff. Siehe auch Schuhen (2002); Pfaffenzeller (2003). Auch ohne Nennung des Governance-Begriffes hat sich die deutschsprachige NPO-Management-Literatur schon früher mit dieser Problematik befasst. Vgl. Schwarz (1996).

12 Vgl. Schwarz (1996), S. 529.

13 Vgl. Cornforth/Simpson (2002), S. 467; Brown (2002), S. 373 ff.; Saidel/Harlan (1998), S. 252 f.

14 Vgl. Schuhen (2002), S. 173.

15 Vgl. Saidel/Harlan (1998), S. 245.

16 Vgl. Murray u.a. (1992), S. $166 \mathrm{f}$. 


\begin{tabular}{ll}
\hline Philanthropic Governance & Corporate Governance \\
\hline Large board size & Small board size \\
\hline Wide range of perspectives/ backgrounds & Narrow, more focused perspectives/ backgrounds \\
\hline Small number of inside directors & Large number of inside directors \\
\hline Separation of management and governance & Active management participation on board \\
\hline Informal management accountability on board & Formal management accountability on board \\
\hline No limit to consecutive terms for board members & Limit on consecutive terms for board members \\
\hline No compensation for board service & Compensation for board service \\
\hline Emphasis on asset and mission preservation & Emphasis on strategic and entrepreneurial activity \\
\hline
\end{tabular}

Tab. 1: Differenzierung zwischen Philanthropic und Corporate Governance

Quelle: $\quad$ Eigene Darstellung in Anlehnung an Alexander/Weiner (1996), S. 225

Bei dieser Differenzierung handelt es sich um zwei Idealtypen, die in der Realität nicht zwingend in reiner Form auftreten. Die sie illustrierenden Merkmale zeigen lediglich zwei gegensätzliche Vorstandsstrukturen mit unterschiedlichen Schwergewichten auf.

\section{Corporate Governance-Prinzipien}

Aufbauend auf den bisherigen Ausführungen werden im Folgenden einige der Best Practice-Prinzipien des Swiss Code of Corporate Governance ${ }^{17}$ mit entsprechenden Gestaltungsempfehlungen der NPO-Management-Lehre verglichen. Im Gegensatz zum Deutschen Corporate Governance Kodex und anderen ausländischen Beispielen enthält der Swiss Code drei Spezifika, die auch im Hinblick auf NPO von Bedeutung sind: ${ }^{18}$ Bereits in der Definition von Corporate Governance wird die Ausrichtung auf StakeholderInteressen, die Betonung der Transparenz sowie die Wahrung der Entscheidungsfähigkeit auf oberster Ebene hervorgehoben. Zudem fällt die Übertragung der schweizerischen Richtlinien auf NPO leichter, da im schweizerischen Recht nur ein einheitliches Leitungsorgan (Verwaltungsrat) der AG und kein zweistufiges System von Aufsichtsrat und Vorstand existiert. ${ }^{19}$ Es werden Gemeinsamkeiten und Unterschiede zwischen Corporate und Nonprofit Governance herausgearbeitet und daraus entsprechende Handlungsempfehlungen für eine wirkungsvolle Nonprofit Governance abgeleitet.

- $\quad$ Eigenständige Willensbildung im kritischen Gedankenaustausch mit der Geschäftsleitung

Der Verwaltungsrat soll durch die personelle Zusammensetzung jene Fähigkeiten in sich vereinigen, die es ihm ermöglichen, die Anträge der Geschäftsleitung kritisch zu hinterfragen und - wo erforderlich - sogar eigenständig Entscheidungen in Abweichung zu den Anträgen zu erarbeiten, um sich dem Completed Staff-work entziehen zu können. Schwarz u.a. postulieren zunächst eine klare Beschränkung der

17 Vgl. Böckli (2002).

18 Vgl. Böckli (2002), S. 3.

19 Vgl. Marti (2002). 
Vorstände auf die politisch-strategischen Entscheidungen mit konsequenter Delegation des Operativen an die Geschäftsführung, eine Aufgabenverteilung, die im Gegensatz zu den Unternehmungen in NPO keineswegs als Selbstverständlichkeit gelten kann..$^{20}$ Denn getreu dem Motto „Management is more fun than policymaking ${ }^{621}$ mischen sich ehrenamtliche Gremien des Öfteren in das operative Geschäft ein, nicht zuletzt als Flucht vor der komplexen strategischen Gedankenarbeit. Für die Erarbeitung der strategischen Entscheide empfiehlt sich - im Sinne des ,interwoven planning" - eine workshopartige Vorgehensweise, die unter Cooperative Governance subsumiert wird: Die Geschäftsleitung hat nicht vollendete Anträge, sondern „offene“ Entscheidungsgrundlagen (Analysen, Alternativen, Bewertungen) zu liefern. Die Eckpunkte der Strategien werden in intensiver Diskussion gemeinsam erarbeitet, anschliessend von der Geschäftsleitung konkretisiert und zum Beschluss vorgelegt. Nur so kann ein ehrenamtliches Gremium seine Steuerungsfunktion tatsächlich wahrnehmen bzw. den politisch-strategischen Gehalt der Entscheidungen substanziell mitprägen. Dazu ist aber ebenfalls eine Auslese qualifizierter Persönlichkeiten für den Vorstand aufgrund von Anforderungsprofilen in einem transparenten Wahlverfahren zu gewährleisten. ${ }^{22}$

- Einführung und Weiterbildung der Verwaltungsräte

Dieses Prinzip gilt 1:1 für Milizgremien, wobei es hier ebenso um NPOspezifisches Management-Know-how (Führung in demokratisch-föderalistischen Strukturen) wie um Sachverstand („Branchenkenntnisse“) geht. Untersuchungen haben gezeigt, dass ehrenamtliche Vorstandsmitglieder aufgrund fehlender Kenntnisse bei der Beurteilung der Management-Arbeit auf eigene berufliche oder persönliche, oft nicht NPO-adäquate Erfahrungen zurückgreifen. ${ }^{23}$ Der Weiterbildung von Ehrenamtlichen wird - nicht zuletzt als Folge der Corporate Governance Forderungen und der damit gesteigerten Verantwortlichkeit der Milizgremien - in Zukunft vermehrte Bedeutung zukommen müssen. ${ }^{24}$

- Rechtzeitige, umfassende und übersichtlich aufbereitete Information des Verwaltungsrates

Dahinter steht eine klare Absage an die offenbar häufig von mit der Entscheidungsvorbereitung betrauten Personen praktizierte „Vernebelungstaktik“. In vielen NPO taktieren die Geschäftsführungen auf analoge Art und Weise, so dass Schwarz u.a. dezidiert „miliztaugliche“ Unterlagen für die Willensbildung und die Kontrolle sowie entsprechend präzise Regelungen in Geschäftsordnungen fordern. ${ }^{25}$

20 Vgl. Schwarz u.a. (2002), S. $97 \mathrm{ff}$.

21 Vgl. Duca (1996).

22 Vgl. Schwarz (2005), S. $252 \mathrm{ff}$.

23 Vgl. Miller (2002), S. 444.

24 Vgl. Holland (2002), S. $420 \mathrm{f}$.

25 Vgl. Schwarz u.a. (2002), S. 110. 
- $\quad$ Übereinstimmung von Finanzen und Strategie

Der Verwaltungsrat hat dafür zu sorgen, ,dass die Gesellschaft keine Strategie einschlägt, die mit den zur Verfügung stehenden Mitteln offensichtlich nicht vereinbar ist ${ }^{\text {‘26. }}$. Auch NPO haben oft mit der Schwierigkeit zu kämpfen, das Wünschbare auf das Machbare, sprich Finanzierbare zu reduzieren. Es empfiehlt sich, auf strategischer Ebene die traditionelle Trias von Zielen, Maßnahmen (hier Strategien) und Mitteln (Ressourcen) anzuwenden bzw. die drei Elemente im strategischen Entscheid in Einklang zu bringen.

- Internes Kontrollsystem mit Risikomanagement und Compliance

Der Verwaltungsrat hat dafür zu sorgen, dass ein internes Kontrollsystem entwickelt und gehandhabt wird, und dass nicht nur die finanziellen, sondern auch die operationellen Risiken durch das Risikomanagement abgedeckt werden. ${ }^{27}$ Diese Forderung ist unmittelbar zu koppeln mit der strategischen Steuerungsfunktion und Oberleitung, die der Verwaltungsrat wahrzunehmen hat.

Für NPO haben Schwarz u.a. ein umfassendes Führungsinstrumenten-System für die Steuerung (Planung und Kontrolle) mit dem Konzept eines die Steuerungsaufgaben unterstützenden Controlling-Systems entwickelt. ${ }^{28}$ Dabei hat der Vorstand mit der Geschäftsleitung zu vereinbaren, welche Informationen ihm in welcher Periodizität und in welcher Form zu liefern sind, damit er seine Kontrollaufgabe intensiv wahrnehmen kann. Dass in einer NPO zwar auch - aber nicht nur - finanzielle Informationen zu Kontrollzwecken aufzubereiten sind, liegt im „Wesen“ dieser Organisationen. Ebenso wichtig - wenn nicht bedeutsamer - ist die Aussagekraft dieser Daten, dieses Reportings in Bezug auf die Erfüllung des NPO-Zweckes, der inhaltlichen, strategischen Ziele, der Nutzenstiftung usw.

Neben diesen weitgehend übereinstimmenden Kriterien enthält der Swiss Code zwei Prinzipien, die für NPO bedenkenswert und bisher kaum thematisiert worden sind:

1. Individuelle Abstimmung bei Verwaltungsratswahl und Dechargeerteilung Dieses Verfahren ermöglicht der Aktionärsversammlung eine individuelle Beurteilung der Ratsmitglieder mit einer allfälligen Sanktionierung ,unerwünschten“ Verhaltens. Globale Wahlen und auch Dechargeerteilungen an das kollegiale Gremium entfallen.

In NPO erfolgt häufig die erstmalige Wahl individuell, aber die Wiederwahl ,in globo“ und häufig auch durch reine Akklamation, da sich die meisten NPO glücklich schätzten, überhaupt genügend Kandidaten oder Kandidatinnen für das Amt gefunden zu haben. Genauso wird bei der Dechargeerteilung verfahren. Über diese Individualisierung gemäss Swiss Code wäre in NPO nachzudenken.

2. Jährliche Besprechung der eigenen Leistung durch den Verwaltungsrat

Dieser Aufforderung zur periodischen Selbstreflexion mit dem Ziel des „,continuous improvement" der Verwaltungsratsarbeit ist gemäß Böckli in der Erarbeitung des 
Swiss Code heftiger Widerstand erwachsen, was kaum verwundern und auch bei Übertragung dieser Massnahme auf NPO-Gremien zutreffen dürfte. Dennoch: Wenn im NPO-Betrieb bis hinauf zur Geschäftsleitung eine jährliche Leistungsbeurteilung durchgeführt wird (als Selbst- und Fremdevaluation), warum sollte sich nicht auch ein Milizgremium dieser Prozedur unterziehen, um sich aufgrund kritischer Auseinandersetzung eigene Entwicklungsziele zu formulieren? ${ }^{29}$ Dabei kommt dem Präsidenten/der Präsidentin eine zentrale Funktion zu, wenn man - wie Carver $^{30}$ - davon ausgeht, dass es nicht Aufgabe von Präsidenten ist, ihre NPO zu führen, sondern sie einzig und allein dafür verantwortlich sind, dass das MilizFührungsgremium seine strategische Steuerungs- und Kontrollaufgabe wirksam und effizient wahrnimmt.

Schließlich sind drei Themen anzuschneiden, in denen die NPO in den meisten Fällen grundlegend von Unternehmungen abweichen:

- Doppelspitze oder Personalunion: Zu einer eindeutigen Antwort konnte sich der Swiss Code offenbar nicht durchringen, so dass beide Formen zugelassen sind: Die Trennung der Ämter „Präsident“ und „CEO“ sowie die Vereinigung der beiden Funktionen in einer Person, wobei ein klares Bekenntnis (ein Beschluss) des Verwaltungsrates zur einen oder anderen Ausprägung erforderlich ist.

In (Größeren) NPO sind zwar in Ausnahmefällen die Figuren des geschäftsleitenden Präsidenten (Doppelmandat) oder diejenige des Geschäftsführers als Vorstandsmitglied anzutreffen. In den weitaus meisten NPO wird jedoch die von Schwarz u.a. zwingend verlangte Gewaltentrennung angewandt, die den Verzicht auf solche Doppelmandate vorsieht. ${ }^{31}$ Präsidien werden als Milizfunktionen angesehen, selbst dann, wenn ein Vorsitzender hauptamtlich tätig ist, neben einem vollamtlich angestellten Geschäftsführer. Je mehr Zeit ein Präsident in sein Amt investiert, desto Größer ist die Gefahr von Konflikten mit der Geschäftsführung im operativen und Interessenvertretungs-Bereich.

- Die Frage der unabhängigen Verwaltungsratsmitglieder: Hier geht es darum, ob und in welchem Ausmass Geschäftsleitungsmitglieder in den Verwaltungsrat Einsitz nehmen können. Gemäss dem Grundsatz der Gewaltentrennung ist auch diese Einsitznahme bei NPO abzulehnen.

- Entschädigungszahlungen: Die Tätigkeit des Führungsorgans einer NPO ist ehrenamtlich. Von Entschädigungszahlungen sollte deshalb im Idealfall abgesehen werden. Die ZEWO-Richtlinien in der Schweiz legen für jedes Vorstandsmitglied fest, dass die ersten 100 Stunden in jedem Fall ehrenamtlich geleistet werden müssen, weiteres Engagement kann entschädigt werden. ${ }^{32}$ Auch wenn Organisationen immer häufiger über Schwierigkeiten berichten, gute Ehrenamtsträger zu gewinnen, ist die

29 Mitglieder in Aufsichtsgremien verschliessen sich dieser Frage gerne mit dem Hinweis, dass sie die Arbeit ja ehrenamtlich machen würden. Vgl. Holland (2002), S. 417 f.

30 Vgl. Carver (1997).

31 Vgl. Schwarz u.a. (2002), S. 173.

32 Vgl. ZEWO (2003), Art. 6 Abs. 1. 
durch das Ehrenamt gewährleistete Unabhängigkeit besonders für die Steuerungsund Überwachungsfunktion des Vorstandes sehr wichtig.

\section{Typenabhängige Governance-Ansätze}

Ein Grossteil der Literatur zu Nonprofit Governance bietet allgemeingültige Lösungen für alle NPO, ohne Berücksichtigung von spezifischen Eigenschaften. ${ }^{33}$ Erst in den letzten Jahren werden sowohl in der normativen als auch empirischen Literatur typenspezifische Governance-Probleme beachtet. ${ }^{34}$

NPO unterscheiden sich in vielfältiger Art und Weise, so z.B. nach Rechtsform, Zweckausrichtung, Finanzierungsarten oder produzierten Gütern. ${ }^{35}$ In Bezug auf Nonprofit Governance sind vor allem die sich aus der Rechtsform ergebenden rechtlichen Bestimmungen, die Finanzierungsarten sowie die Zweckausrichtung von Interesse.

Im Allgemeinen können sich NPO als Verein, Stiftung oder Genossenschaft konstituieren, ${ }^{36}$ wobei die jeweiligen Gesetzesvorschriften mehr oder weniger präzise Aussagen zu Gestaltung, Struktur und Arbeitsweise des ehrenamtlichen Führungsgremiums machen. Im Hinblick auf die Zweckausrichtung lassen sich Eigenleistungs- und DrittleistungsNPO unterscheiden. Eigenleistungs-NPO erbringen vornehmlich Leistungen für die eigenen Mitglieder (Identitätsprinzip), Drittleistungs-NPO setzen sich für die Belange einer spezifischen Gruppe ein. ${ }^{37}$

\section{Nonprofit Governance in Drittleistungs-NPO}

Der überwiegenden Mehrheit amerikanischer Beiträge zur Nonprofit GovernanceForschung liegen Samples mit Krankenhäusern, ${ }^{38}$ spenden-orientierten $\mathrm{NPO}^{39}$ und Wohlfahrts- oder Freiwilligen-Organisation zugrunde.$^{40}$ Es handelt sich also vorwiegend um Drittleistungs-NPO. Besonders deutlich wird dies an der Studie von Abzug/Galaskiewicz, die fünfzehn verschiedene Typen von NPO untersuchen, die aber alle Drittleistungs-NPO sind. $^{41}$

Es verwundert daher nicht, dass der Resource Dependency-Ansatz die dominante theoretische Grundlage für Nonprofit Governance-Modelle ist. ${ }^{42}$ Die wichtigste Aufgabe des Boards ist es gemäss diesem Ansatz, die Überlebensfähigkeit der Organisation zu si-

33 Vgl. Houle (1997); Carver (1997); Alexander/Weiner (1996).

34 Vgl. Schuhen (2002); Friedman/Philipps (2004).

35 Vgl. Schwarz u.a. (2002), S. 20 ff.

36 Die gGmbH soll hier als stark profit-orientierte Rechtsform ausgeklammert werden.

37 Vgl. Purtschert (2001), S. $51 \mathrm{f}$.

38 Vgl. Alexander/Weiner (1996); Eldenburg/Krishnan (2003); Eldenburg u.a. (2003).

39 Vgl. Callen u.a. (2003);

40 Vgl. Bailey (1992); Brown/Iverson (2004); Miller (2002); Murray u.a. (1992); Preston/Brown (2004).

41 Vgl. Abzug/Galaskiewicz (2001), S. 56.

42 Vgl. Miller-Millesen (2003), S. 533. Siehe auch Fletcher (1992); Harlan/Saidel (1994); Jun/Armstrong (1997); Middelton (1987); Pfeffer (1973). 
chern, indem es stabile Beziehungen zur Umwelt - besonders zu Ressourcengebern aufbaut und pflegt. ${ }^{43}$

Harlan/Saidel entwickeln vier Rollen, mit denen Boards die Beziehung zwischen der NPO und dem Staat als wichtigem Finanzgeber einnehmen: ${ }^{44}$ faciliator role, political advocate role, buffer role, values guardian role. ${ }^{45}$ Dabei ist das Board aber auf die $\mathrm{Zu}$ sammenarbeit mit den hauptamtlichen Mitarbeitern angewiesen und in manchen Governance-Aktivitäten ist die Geschäftsführung sogar aktiver involviert als das Board selbst. ${ }^{46}$ Das Ausmaß der ressourcen-orientierten Aktivitäten des Boards hängt dabei eng mit der Notwendigkeit der externen Geld- und Ressourcenbeschaffung für die Organisation zusammen. ${ }^{47}$ Aus dieser Feststellung lässt sich folgern, dass die Relevanz der RessourcenOrientierung abnimmt, je geringer die Abhängigkeit der Organisation von der finanziellen Unterstützung externer Anspruchsgruppen ist, bzw. je intensiver die Finanzleistungen vertraglich oder gesetzlich geregelt sind (etwas durch Leistungsvertrag zwischen Staat und NPO).

Basierend auf einer umfangreichen quantitativen Erhebung, ${ }^{48}$ hat Schuhen ein Governance-Modell der freien Wohlfahrtspflege entwickelt. ${ }^{49}$ Sein Ansatz stützt sich v.a. auf die Ergebnisse der obengenannten Studie von Alexander/Weiner, ${ }^{50}$ weswegen auch hier Krankenhäuser als Untersuchungsgegenstand gewählt wurden. Ausgehend von der Beobachtung, dass in NPO meistens Mischformen zwischen Corporate und Philanthropic Governance bestehen, werden die beiden Ansätze zu einer hybriden Steuerung zusammengefasst, die zum einen traditionelle, wertorientierte Bestandteile, zum anderen moderne, kontraktorientierte Steuerungselemente enthält. In der Privatwirtschaft kann die Organisationskultur die Organisationsstruktur stabilisieren (wertorientierter Ansatz), bei NPO ist jedoch das Gegenteil der Fall: „Die weltanschaulich geprägte und durch Leitbilder präzisierte Organisationskultur muss durch die Definition von Vertragsbeziehungen und eindeutigen Aufgabenbeschreibungen und -abgrenzungen stabilisiert werden (kontraktorientierter Ansatz). ${ }^{\text {"51 }}$ Das Ehrenamtsgremium hat dabei die Zielsetzungsverantwortung, während das Management die Durchführungsverantwortung übernimmt. ${ }^{52}$

43 Vgl. Herzlinger (1998), S. 26.

44 Die Autoren definieren organisationelle Unsicherheit vor allem als finanzielle, einnahmenbezogene Unsicherheit. Vgl. Harlan/Saidel (1994), S. 176.

45 Vgl. Harlan/Saidel (1994), S. 175 f.

46 Vgl. Saidel/Harlan (1998), S. 255.

47 Vgl. Miller-Millesen (2003), S. 535.

48 Die Gesamtumfang der Stichprobe betrug $n=543$ Trägerorganisationen bei einem Rücklauf von ca. $27 \%$ oder 144 Antworten.

49 Vgl. Schuhen (2002), S. 134 ff.

50 Vgl. Alexander/Weiner (1996).

51 Vgl. Schuhen (2004), S. 23 f.

52 In diesem Punkt stimmt Schuhen weitestgehend mit Carver (1997) überein, der auch eine klare Aufgabentrennung von Board und CEO empfiehlt. 


\begin{tabular}{|c|c|}
\hline $\begin{array}{l}\text { Elemente wertorientierter/traditioneller Steuerung } \\
\text { (Philantrophic Governance) }\end{array}$ & $\begin{array}{l}\text { Elemente kontraktorientierter/moderner Steuerung } \\
\text { (Corporate Governance) }\end{array}$ \\
\hline Leitung durch Leitbilder & Leitung durch Zielvereinbarungen \\
\hline $\begin{array}{l}\text { Rekrutierung von Leitungskräften und Mitarbeitern } \\
\text { primär nach deren Wertorientierung }\end{array}$ & $\begin{array}{l}\text { Rekrutierung von Leitungskräften und Mitarbeitern } \\
\text { primär nach fachlichen Gesichtspunkten }\end{array}$ \\
\hline Unklare oder keine Aufgabenbeschreibungen & Konkrete Aufgabenbeschreibungen \\
\hline Dominanz ehrenamtlicher Leitungskräfte & Dominanz hauptamtlicher Leitungskräfte \\
\hline $\begin{array}{l}\text { Lose/unklare (Vertrags-)Beziehungen zum Verband } \\
\text { und zwischen Träger und Einrichtungen/ Diensten }\end{array}$ & $\begin{array}{l}\text { Feste/klare (Vertrags-)Beziehungen zum Verband und } \\
\text { zwischen Träger und Einrichtungen/Diensten }\end{array}$ \\
\hline $\begin{array}{l}\text { Vermischung von Governance- und Management- } \\
\text { aufgaben }\end{array}$ & Trennung von Governance- und Managementaufgaben \\
\hline Vertrauensbasierte Steuerung & Informationsbasierte Steuerung \\
\hline \multicolumn{2}{|c|}{$\begin{array}{l}\text { Hybride Steuerung als Mix von Merkmalen aus beiden Typen: } \\
\text { Das Governance-Modell der Freien Wohlfahrtspflege }\end{array}$} \\
\hline
\end{tabular}

\section{Tab. 2: Das Nonprofit Governance-Modell der freien Wohlfahrtspflege}

Quelle: $\quad$ Eigene Darstellung in Anlehnung an Schuhen (2002), S. 164

Für die Umsetzung des Modells werden Massnahmen zu Board Development und Board Assessment ${ }^{53}$ sowie Management- und Führungsinstrumente wie zielorientierte Steuerung (Governance by Objectives), Risiko- und Qualitätsmanagement ${ }^{54}$ oder der Abschluss franchiseartiger Kontrakte ${ }^{55}$ zwischen Träger und operativer Einheit besprochen. $^{56}$

Im Gegensatz zu früheren quantitativ-empirischen Nonprofit Governance-Modellen ${ }^{57}$ tragen die bisher vorgestellten Ansätze bereits der Erkenntnis Rechnung, dass es nicht einen besten Weg gibt, sondern in Abhängigkeit von Rechtsform, Zweckausrichtung, Organisationskultur und anderen Parametern die Governance-Struktur an die Organisation angepasst werden muss. Trotzdem liegen diesen Ansätzen Annahmen zugrunde, die hauptsächlich auf Drittleistungs-NPO ausgerichtet sind und sich nicht ohne weiteres auf Eigenleistungs-NPO übertragen lassen.

Ein weiterer Ansatz ist das Governance-Netzwerk von Pfaffenzeller. Es berücksichtigt aus institutionen-ökonomischer Sicht alle Stakeholder, die wertvolle Investitionen getätigt haben und deshalb in einer Machtbeziehung zur NPO stehen. ${ }^{58}$ Die Machtbeziehungen müssen getrennt voneinander betrachtet und analysiert werden. Die spezifischen Investitionen der einzelnen Stakeholdergruppen sind v.a. Zeit und finanzielle Mittel. Bei der Gruppe der Kunden wird davon ausgegangen, dass sie die empfangenen Leistungen

53 Vgl. Holland (1991), S. 25 ff.

54 Vgl. Purtschert/von Schnurbein (2004), S. 323 ff.

55 Vgl. Schuhen (2002), S. 181 ff. Unter Social Franchising versteht man „die nicht profit-orientierte Form vertikaler Kooperation zwischen gleichberechtigten Partnern im Sozialbereich zur Replikation sozialer Programme und Dienstleistungen sowie Steuerung und Vernetzung sozialer Organisationen.“ Schuhen (2000), S. 29.

56 Vgl. Schuhen (2004), S. 25.

57 Vgl. Schuhen (2002), S. 108.

58 Vgl. Pfaffenzeller (2003), S. 161. Das Modell beruht auf der Definition nach Rajan/Zingales, wonach ein Individuum über Macht verfügt, wenn es wertvolle Ressourcen in einen Prozess einbringt und kontrolliert. Vgl. Rajan/Zingales (2000), S. 4. 
bezahlen oder, falls diese unentgeltlich sind, die Macht der Kunden über die Wahrnehmung von Konkurrenzangeboten am Markt entsteht (Abwanderung). ${ }^{59}$

Im Zentrum der Netzwerkstruktur steht das Management, d.h. die Geschäftsführung, da es als einziger Akteur mit allen anderen Stakeholdern explizite oder implizite (unvollständige) Verträge abschliesst. ${ }^{60}$ Aufgabe des Managements ist es nun, ausgleichend auf die Machtverhältnisse zu wirken und die Verteilung der Quasi-Renten zu steuern. Besonders hervorgehoben werden dabei die Key-Stakeholder „Spender“ und „freiwillige Mitarbeiter", da sie ihre Quasi-Renten nicht durch eine besondere Machstellung schützen können. Durch eine Reihe von formalen und informalen Governance-Instrumenten müssen diese Anspruchsgruppen besonders berücksichtigt und geschützt werden. ${ }^{61}$

Das Governance-Netzwerk-Modell weist einen sehr hohen Detaillierungsgrad auf und legt der Nonprofit Governance eine sehr breite Basis der Organisationsstruktur zugrunde. Die dem Modell vorangestellte Definition von NPO erfasst aber v.a. Drittleistungs-NPO. Deshalb werden die Mitglieder explizit nicht als eigene Stakeholder-Gruppe angesehen, sondern in andere Gruppen wie Gründer, Spender oder ehrenamtliche Mitarbeiter subsumiert. $^{62}$

Die Erklärung von Governance-Strukuren anhand der Machtverteilung entspricht zweifellos in vielerlei Hinsicht der Realität von NPO. Jedoch ist die Machtstruktur in NPO nicht nur auf die Ressourcenverteilung zurückzuführen. Daneben existieren formale und informale Machtquellen, die ebenfalls zu berücksichtigen sind. ${ }^{63}$ Das Board wird als eine von vielen Anspruchgruppen behandelt, was dem generellen Verständnis widerspricht, dass das Board neben dem Management für die Governance verantwortlich ist. ${ }^{64}$ Außerdem besteht in diesem Modell die Gefahr, dass dem Management eine zu grosse Machtfülle eingeräumt wird, und das Board letztendlich zu einem Absegnungs-Komitee verkommt. ${ }^{65}$ Weiterhin nicht beachtet werden mögliche Beziehungen und Austauschprozesse zwischen den einzelnen Stakeholder-Gruppen. Die getrennte Betrachtungsweise kann dazu führen, dass wichtige Aspekte des Netzwerkes übersehen werden. Auch die Tatsache, dass die gleichen Individuen mehreren Anspruchgruppen zuzurechnen sind (z.B. die Mitglieder oder Board-Mitglieder, die zugleich Spender, Vertreter der Dachorganisation oder der staatlichen Behörden sein können), muss bei der Ausgestaltung des Netzwerkes berücksichtigt werden.

Diese auf Drittleistungs-NPO fokussierten Ansätze zeigen, dass zwar typenspezifisch einzelne Governance-Elemente angepasst, und somit das Grundmodell teilweise variiert werden muss, dass jedoch - wie anhand des Swiss Code exemplifiziert - einige Basisprinzipien unantastbar bleiben, wenn von Nonprofit Governance als einem bestimmten Gestaltungsmodell und nicht einfach von einem Management-/Führungsmodell gespro-

59 Vgl. Hirschmann (1974).

60 Vgl. Pfaffenzeller (2003), S. 162. Diese Sichtweise wird auch von Herman/Heimovicz (1991) und Chait u.a. (1996) vertreten.

61 Vgl. Pfaffenzeller (2003), S. 184 ff.

62 Vgl. Pfaffenzeller (2003), S. 167.

63 Vgl. Pfaffenzeller (2003), S. 153.

64 Vgl. Saidel/Harlan (1998), S. 244. Siehe auch Golensky (1993), S. 184 ff.

65 Vgl. Cornforth (2002), S. 10. 
chen werden soll. So sind insbesondere jene Regeln als unverzichtbar zu betrachten, welche oben aus dem Swiss Code abgeleitet und als zentrale Prinzipien des Zusammenwirkens von Geschäftsführung und Vorstand postuliert werden. Dies gilt im gleichen Masse für die nachstehend beschriebenen auf Eigenleistungs-NPO bezogene Ansätze.

\section{Nonprofit Governance in Eigenleistungs-NPO}

Im Gegensatz zu den bisher genannten Nonprofit Governance-Modellen betrachtet das Cupped Hands Model von Friedman/Phillips nicht Drittleistungs-NPO, sondern Berufsverbände, also Eigenleistungs-NPO. ${ }^{66}$ Die Führungsproblematik dieser Organisationen schildern die Autoren folgendermaßen:

,...the traditional model of running professional associations is premised on the high involvement of volunteers who establish, and often implement, policy through large councils and complex committee structures that meet frequently. This results in ponderous, ritualized procedures that hinder decision making and are unsuited to a complex and rapidly changing environment. ",67

Drei Probleme finden sich in fast allen Eigenleistungs-NPO wieder:

- $\quad$ eine personelle Überbesetzung des Vorstandes (Anzahl der Vorstandsmitglieder)

- $\quad$ eine von Repräsentation und nicht von Erfahrung und Wissen geprägte Zusammensetzung des Vorstandes sowie

- $\quad$ eine Zweiteilung in einen kleinen und einen grossen Vorstand, wobei die Entscheidbefugnis teilweise auf den kleinen Vorstand übertragen wird, die rechtliche Verantwortung jedoch beim großen Vorstand verbleibt. ${ }^{68}$

Um zu verhindern, dass der Vorstand nicht als eine zu überwindende Hürde angesehen wird, sondern einen Mehrwert für die Organisation schafft, werden die Aufgaben der einzelnen Akteure klar getrennt. ${ }^{69}$ In Abbildung 1 ist die Funktionsweise des Modells schematisch dargestellt. Die „linken“ Hände entwickeln Ziele und Pläne für die ,inneren“ Akteure, während die „rechten“ Hände den ,inneren“ Akteur auf diese Ziele und Pläne hin kontrollieren und evaluieren.

66 Vgl. Friedman/Philipps (2004), S. 187.

67 Friedman/Philipps (2004), S. 188.

68 Vgl. Wood (1992), S. 148.

69 Die Autoren benennen vier Akteure: CEO, eine Strategie-Gruppe, eine Repräsentations-Gruppe und die Mitarbeiter. Vgl. Friedman/Philipps (2004), S. 195. 


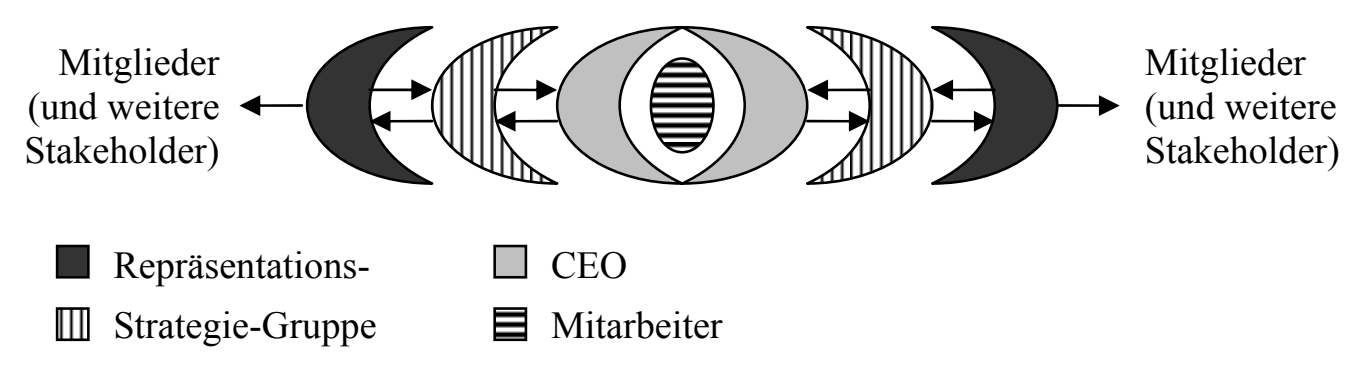

\section{Abb. 1: Das Cupped Hands Model}

Quelle: $\quad$ Eigene Darstellung in Anlehnung an Friedman/Philipps (2004), S. 196

Die Aufgabe der Repräsentations-Gruppe besteht darin, die Interessen und Wünsche der Mitglieder zu erfassen und gegenüber der Strategie-Gruppe zu vertreten. Anschließend muss sie die Umsetzung überwachen und schließlich ihre Bewertung an die Mitglieder zurückgeben. Die Strategie-Gruppe wiederum erfüllt die gleichen Aufgaben gegenüber dem CEO, der sie wiederum auf die Mitarbeiter überträgt. Diesem System von Informationsströmen liegen sechs Prinzipien zugrunde: Relevanz, Zuverlässigkeit, Vergleichbarkeit, Verständlichkeit, Rechtzeitigkeit und Interaktion. ${ }^{70}$

Unter den wenigen angloamerikanischen Beiträgen zur Governance in EigenleistungsNPO kann das Cupped Hands Model als origineller Ansatz gewertet werden, der aber zahlreiche Fragen offen lässt.

Die Autoren schränken selbst bereits ein, dass die linearen Kommunikationsströme die Realität vereinfacht darstellen und bspw. der Austausch mit den Mitgliedern nicht der Repräsentations-Gruppe vorbehalten ist. Strategien können auch auf „wilden“, emergenten Kommunikationspfaden erfolgreich entstehen und umgesetzt werden. Eine weitere Schwäche des Modells liegt in der mangelnden Berücksichtigung der Verantwortlichkeit. Das Cupped Hands Model gibt keine Antwort auf die Frage, wer wie viel Verantwortung zu tragen hat sowie wo und wie strategische Entscheide letztendlich gefällt werden. Ebenso fehlt eine Erklärung, von wem die Repräsentations-Gruppe eingesetzt bzw. gewählt wird.

Als eine präzisere Antwort auf diese Fragen und damit aussagekräftiger für die Governance-Problematik kann der Principal-Agent-Ansatz ${ }^{71}$ gewertet werden. Er ist auf alle Beziehungen zwischen über- und nachgeordneten Instanzen anwendbar, entlang der gesamten Verbandshierarchie, von der Mitlgieder-/Delegiertenversammlung (als obersten Prinzipal) bin hinab zu den Mitarbeitenden (Agenten). Auch dem grundsätzlich für alle NPO-Typen geltenden Policy Governance Model ${ }^{72}$ kommt eine höhere Erklärungs- und Gestaltungskraft zu.

70 Vgl. Friedman/Philipps (2004), S. 198 f.

71 Vgl. Jensen/Meckling (1976).

72 Vgl. Carver (1997). 
Abgesehen vom Cupped Hands Model sind bisher im anglo-amerikanischen Raum kaum weitere Versuche unternommen worden, einen Governance-Ansatz auf die Bedürfnisse und Herausforderungen von Eigenleistungs-NPO zuzuschneiden, obwohl die Verschiedenartigkeit von NPO-Typen generell bekannt ist. ${ }^{73}$

Im deutschsprachigen Raum hat die NPO-Managementlehre - sofern sie ausdrücklich auch auf Verbände fokussiert ist - wesentliche Erkenntnisse zur Erklärung und Gestaltung der Nonprofit Governance in Eigenleistungs-NPO erarbeitet. Ein typisches Beispiel (unter anderem) ist das Freiburger Management-Modell für NPO. ${ }^{74}$

\section{Zusammenfassende Bemerkungen}

Der für Profitorganisationen entwickelte Corporate Governance-Ansatz ist zu einem überwiegenden Teil auf NPO übertragbar, weil die Situation und Konstellationen weitgehend ähnlich sind. Der Erfolg von Good Practice misst sich jedoch in NPO nicht am Shareholder-Value, sondern am Mitglieder- und Klientennutzen sowie am Grad der Akzeptanz bei den übrigen Austauschpartnern (wie Staat, Spenderinnen und Spender, Medien, andere Organisationen). In Strukturen mit einer hauptamtlichen Geschäftsführung sollte sich der Vorstand/Stiftungsrat aus Sicht einer wirkungsvollen Nonprofit Governance deshalb auf seine Rolle als Steuerungsgremium auf strategischer Ebene beschränken und der Versuchung widerstehen, ins operative Geschäft einzugreifen. Der Tätigkeitsbereich des Führungsgremiums erstreckt sich v.a. auf Policy Making und Setting Limits sowie auf die Fortschritts- und Ergebniskontrolle. Aus Sicht einer effizienten und effektiven Führung der Organisation sollten Ehrenamtliche und hauptamtliche Mitarbeiter gemeinsam Mittel und Wege zu einer funktionierenden Aufgabenteilung und Zusammenarbeit finden. $\mathrm{Zu}$ diesem Zweck sind die folgenden Zielsetzungen anzustreben und zu realisieren:

- Klare Abgrenzung der Verantwortungs- und Aufgabenbereiche

- Wirkungsvolle Nutzung der beschränkten Kapazitäten (Zeit, Sachverstand, Informationen) der ehrenamtlichen Mitarbeiter

- Gleichgewichtiges Zusammenwirken der beiden Parteien

- Beschränkung der Tätigkeiten der ehrenamtlichen Führungsorgane auf das Wesentliche

- Partizipatives Erarbeiten der Grundsätze, Schwerpunkte der Rahmenentscheide zwischen Ehrenamt und Hauptamt

- Gemeinsame Wahrnehmung der „unteilbaren Führungs-/Problemlösungs-Verantwortung" für die Organisation insgesamt

- Beachtung der Interessen und Einflüsse der einzelnen Anspruchsgruppen.

73 Zu erwähnen sind hier zwei Studien von Young u.a. (1996) und Young (2002) zu nationalen Dachverbänden, die sich aber mehr mit der Struktur der Organisationen als mit Governance-Problemen auseinandersetzen.

Vgl. Schwarz u.a. (2002). 
Es sei nicht verhehlt, dass diese Zielsetzung sehr hoch gesteckt ist, und viele NPO erst auf dem Wege sind oder sich auf den Weg machen, um diesen Anforderungen der Nonprofit Governance (schrittweise, in Lernprozessen) gerecht $\mathrm{zu}$ werden. In der Schweiz beweisen jedoch die von NPO ins Leben gerufenen Initiativen - wie etwa der Swiss Code of Best Foundation Governance ${ }^{75}$ - dass sich die Organisationen ernsthaft mit der Thematik beschäftigen, weil sie die zwingende Notwendigkeit wirkungsvoller und effizienter Governance-Strukturen als wesentliches Element ihrer Überlebens- und Fortschrittsfähigkeit erkannt haben.

\begin{abstract}
Peter Schwarz and Georg von Schnurbein, Similarities and structural differences of corporate and nonprofit governance
\end{abstract}

Board Structure; Completed Staff-work; Corporate Governance; Nonprofit Governance; Type-related Governance Approaches; Trustees

The different management and organisational structure in nonprofit organisations require adapted governance means. This article compares some best practice principles of the Swiss Code of Corporate Governance with the corresponding recommendations of nonprofit management literature. Thereby, the similarities and differences of corporate and nonprofit governance will be described, as well as governance problems of different npo-types. Finally, some guidelines for effective nonprofit governance will be presented.

\title{
Literaturverzeichnis
}

Abzug, Rita und Joseph Galaskiewicz (2001), Nonprofit Boards: Crucibles of Expertise or Symbols of Local Identities?, in: Nonprofit and Voluntary Sector Quarterly, Vol. 30, Nr. 1, S. 51-73

Alexander, Jeffery. A. und Bryan J. Weiner (1996), The Adoption of the Corporate Governance Model by Nonprofit Organizations, in: Nonprofit Management \& Leadership, Vol. 8, Nr. 3, S. 223-242

Bailey, Darlyne (1992), The Strategic Restructering of Nonprofit Associations: An Exploratory Study, in: Nonprofit Management \& Leadership, Vol. 3, Nr. 1, S. 65-80

Böckli, Peter (2002), Harte Stellen im Soft Law, in: Der Schweizer Treuhänder, Heft 11, S. 1-12

Bright Preston, Jennifer und William A. Brown (2004), Commitment and Performance of Nonprofit Board Members, in: Nonprofit Management \& Leadership, Vol. 15, Nr. 2, S. 221-238

Brönnimann, Thomas (2003), Corporate Governance und die Organisation des Verwaltungsrates, Bern, Stuttgart und Wien

Brown, William A. (2002), Inclusive Governance Practices in Nonprofit Organizations and Implications for Practice, in: Nonprofit Management and Leadership, Vol. 12, Nr. 4, S. 369-386.

Brown, William A. und Joel O. Iverson (2004), Exploring Strategy and Board Structure in Nonprofit Organizations, in: Nonprofit and Voluntary Sector Quarterly, Vol. 33, Nr. 3, S. 377-400

Cadbury, Adrian Sir (1992), The Cadbury Committee Report: Financial Aspects of Corporate Governance, London

Callen, Jeffrey L., April Klein und Daniel Tinkelman (2003), Board Composition, Committees, and Organizational Efficiency: The Case of Nonprofits, in: Nonprofit and Voluntary Sector Quarterly, Vol. 32, Nr. 4, S. 493-520

Carver, John (1990), Boards That Make a Difference, San Francisco

Carver, John (1997), Boards That Make a Difference, 2. Aufl., San Francisco

75 Vgl. Egger (2005). 
Chait, Richard, Thomas Holland und Barbara Taylor (1996), The New Work of the Nonprofit Board, in: Harvard Business Review, Sept/Oct, S. 36-46

Cornforth, Chris (Hrsg.) (2002), The Governance of Public and Nonprofit Organisations: What Do Boards Do?, London

Cornforth, Chris and Claire Simpson (2002), Change and Continuity in the Governance of Nonprofit Organizations in the United Kingdom, in: Nonprofit Management \& Leadership, Vol. 12, Nr. 4, S. 451-470

Drucker, Peter F. (1990), Lessons for Successful Nonprofit Governance, in: Nonprofit Management \& Leadership, Vol. 1, Nr. 1, S. 7-14

Duca, Diane J. (1996), Nonprofit Boards. Roles, Responsibilities and Performance, New York

Egger, Philipp (2005) (Hrsg.), Swiss Code of Best Foundation Practice, Basel

Eldenburg, Leslie und Ranjani Krishnan (2003), Public versus Private Governance: A Study of Incentives and Operational Performance, in: Journal of Accounting and Economics, o. Jg., Nr. 35, S. 377-404

Eldenburg, Leslie, u.a. (2003), Governance, Performance Objectives and Organizational Form: Evidence from Hospitals, in: Journal of Corporate Finance, o. Jg., Nr. 191, S. 1-22

Fletcher, Kathleen B. (1992), Effective Boards: How Executive Directors Define and Develop Them, in: Nonprofit Management \& Leadership, Vol. 2, Nr. 3, S. 283-293

Friedman, Andrew und Mary Philipps (2004), Balancing Strategy and Accountability, in: Nonprofit Management \& Leadership, Vol. 15, Nr. 2, S. 187-204

Golensky, Martha (1993), The Board Executive Relationship in Nonprofit Organizations: Partnership or Power Struggle?, in: Nonprofit Management \& Leadership, Vol. 4, Nr. 2, S. 177-191

Harlan, Sharon L. und Judith R. Saidel (1994), Board Members Influence on the Government-Nonprofit Relationship, in: Nonprofit and Management Leadership, Vol. 5, Nr. 2, S. 173-196

Herman, Robert D. und Richard Heimovics (1990), The Effective Nonprofit Executive: Leader of the Board, in: Nonprofit Management \& Leadership, Vol. 1, Nr. 2, S. 167-180

Herzlinger, Regina (1998), Wirkungsvolle Aufsicht - Eine Anleitung für Vorstandsmitglieder von NonprofitOrganisationen, in: Verbands-Management, Nr. 1/98, S. 24-36

Hirschmann, Albert O. (1974): Abwanderung und Widerspruch, Tübingen

Holland, Thomas P. (1991), Self-Assessment by Nonprofit Boards, in: Nonprofit Management \& Leadership, Vol. 2, Nr. 1, S. 25-36

Holland, Thomas P. (2002), Board Accountability, in: Nonprofit Management \& Leadership, Vol. 12, Nr. 4, S. 409428

Houle, Cyril O. (1997), Governing Boards, San Francisco

Jensen, Michael und William Meckling (1976), Theory of the Firm - Managerial Behaviour, Agency Costs and Ownership Structure, in: Journal of Financial Economics, o. Jg., Nr. 3, S. 305-360

Jun, Sung P. und Gordon M. Armstrong (1997), The Basis of Power in Churches - An Analysis from a Resource Dependence Perspective, in: Social Science Journal, Nr. 34, S. 105-125

Marti, Mario (2002), Corporate Governance in der Schweiz - Vergleich mit den Regeln in Deutschland, in: Mitteilungen zum deutsch-schweizerischen Rechtsverkehr, August 2002, o.S.

Middleton, Melissa (1987), Nonprofit Board of Directors; Beyond the Governance Function, in: The Nonprofit Sector: A Research Handbook, hrsg. von Walter W. Powell, New Haven, S. 141-153

Miller, Judith, L. (2002), The Board as a Monitor of Organizational Activity, in: Nonprofit Management \& Leadership, Vol. 12, Nr. 4, S. 429-450

Miller-Millesen, Judith L. (2003), Understanding the Behavior of Nonprofit Boards of Directors: A Theory-Based Approach, in: Nonprofit and Voluntary Sector Quarterly, Vol. 32, Nr. 4, S. 521-547

Murray, Vic, Patricia Bradshaw und Jacob Wolpin (1992), Power in and Around Nonprofit Boards - A Neglected Dimension of Governance, in: Nonprofit Management \& Leadership, Vol. 3, Nr. 2, S. 165-182

Pfaffenzeller, Herwig (2003), Die Governance von Nonprofit Organisationen - ein institutionenökonomischer Ansatz, Wien

Pfeffer, Jeffrey (1973), Size, Composition, and Function of Hospital Boards of Directors - A Study of Organizationenviroment Linkage, in: Administrative Science Quarterly, Nr. 18, S. 349-364

Powell, Walter W. (Hrsg.) (1987), The Nonprofit Sector: A Research Handbook, New Haven

Purtschert, Robert (2001), Marketing für Verbände und weitere Nonprofit-Organisationen, Bern, Stuttgart und Wien

Purtschert, Robert und Georg v. Schnurbein (2004), Das NPO-Label für Management-Excellence als Instrument der Corporate Governance, in: Gutes besser tun - Corporate Governance in Nonprofit Organisationen, hrsg. von Ruth Voggensperger u.a., Bern, Stuttgart und Wien, S. 323-342

Rajan, Raghuram G. und Luigi Zingales (2000), The Governance of the New Enterprise, NBER Paper No. 7958

Saidel, Judith, R. und Sharon L. Harlan (1998), Contracting and Patterns of Nonprofit Governance, in: Nonprofit Management \& Leadership, Vol. 8, Nr. 3, S. 243-259

Schauer, Reinbert (2003), Rechnungswesen für Nonprofit-Organisationen, 2. Aufl. Bern, Stuttgart und Wien

Schuhen, Axel (2000), Marke Caritas zusammenhalten - Social Franchising als Modell zur Steuerung der Beziehung innerhalb eines Wohlfahrtsverbandes verbindet lokale Autonomie und Verbandsidentität, in: Neue Caritas, 101. Jg., Nr. 12, S. 28-31

Schuhen, Axel (2002), Das Nonprofit Governance Modell in der Freien Wohlfahrtspflege, Baden-Baden 
Schuhen, Axel (2004), Nonprofit Governance in der Freien Wohlfahrtspflege, in: Verbands-Management, o. Jg., Nr. 3, S. 18-31

Schwarz, Peter (1996), Management in Nonprofit-Organisationen, 2. Auflage, Bern, Stuttgart und Wien

Schwarz, Peter (2005), Organisation in Nonprofit-Organisationen - Grundlagen, Strukturen, Bern, Stuttgart und Wien

Schwarz, Peter u.a. (2002), Das Freiburger Management-Modell für Nonprofit-Organisationen, 4. Auflage, Bern, Stuttgart und Wien

Seibel, Wolfgang (1992), Funktionaler Dilettantismus, Erfolgreich scheiternde Organisationen im „Dritten Sektor” zwischen Markt und Staat, Baden-Baden

Vierheller, Rainer (1983), Demokratie und Management, Göttingen

Voggensperger, Ruth u.a. (Hrsg.) (2004), Gutes besser tun - Corporate Governance in Nonprofit Organisationen, Bern, Stuttgart und Wien

Wood, Miriam M. (1992), Is Governing Board Behavior Cyclical?, in: Nonprofit Management \& Leadership, Vol. 3, Nr. 2, S. 139-163

Young, Dennis R. (2002), The Influence of Business on Nonprofit Organizations and the Complexity of Nonprofit Accountability, in: American Review of Public Administration, Vol. 32, Nr. 1, S. 3-19

Young, Dennis R., Neil Bania und Darlyne Bailey (1996), Structure and Accountability - A Study of National Nonprofit Associations, in: Nonprofit Management \& Leadership, Vol. 6, Nr. 4, S. 347-365

ZEWO (Hrsg.) (2003), Reglement über das ZEWO-Gütesiegel für gemeinnützige Organisationen, Zürich 